\title{
Dual isotope and multidetector camera: The best choices for a specific end-point
}

\author{
Roberta Assante, MD, a and Wanda Acampa, MD, PhD ${ }^{\mathrm{a}, \mathrm{b}}$ \\ ${ }^{a}$ Department of Advanced Biomedical Sciences, University Federico II, Naples, Italy \\ ${ }^{b}$ Institute of Biostructure and Bioimaging, National Council of Research, Naples, Italy
}

Received Apr 12, 2016; accepted Apr 12, 2016

doi:10.1007/s12350-016-0520-5

\section{See related article, pp. $1361-1369$}

Assessment of myocardial infarct tissue has shown to play an important role in risk-stratify patients for adverse cardiac events. ${ }^{1}$ Infarct tissue may show considerable spatial heterogeneity due to the presence of necrotic areas that are intermingled with bundles of viable cardiomyocytes. Myocardial necrosis is primarily located in the middle of the infarct region, while the regions of infarct tissue heterogeneity are primarily present in the periphery of the infarcted myocardium, which is commonly referred as infarct border zone or peri-infarct region. Peri-infarct region is an important substrate for the development of ventricular arrhythmias through the presence of reentrant circuits originating from slow-conduction pathways. ${ }^{2}$ Different imaging modalities have been used for the characterization of both infarct and peri-infarct regions. Myocardial perfusion imaging (MPI) techniques have been widely used in the evaluation of infarct tissue after myocardial infarction. $^{3}$ As well, myocardial imaging with 123Imetaiodobenzylguanidine (MIBG) is a recognized imaging modality for non-invasive assessment of global and regional cardiac sympathetic innervation related to heart failure. ${ }^{4,5}$ The association of myocardial perfusion and innervation imaging has been used in patients after myocardial infarction as well in patients with hypertrophic and dilated cardiomyopathy. ${ }^{6,7}$ In particular, the combined use of these two different imaging modalities provided useful informations in identifying the presence

Reprint requests: Wanda Acampa, MD, PhD, Department of Advanced Biomedical Sciences, University Federico II, Via Pansini 5, 80131 Naples, Italy; acampa@unina.it

J Nucl Cardiol 2017;24:1370-3.

1071-3581/\$34.00

Copyright (C) 2016 American Society of Nuclear Cardiology. of viable but sympathetically denervated myocardial areas, defined as trigger zone. In fact, myocardial 123IMIBG uptake was not only reduced in the central infarct zone involved by the severe reduction of perfusion tracer uptake, but also in adjacent still viable regions as well as in areas with acute and chronic ischemia reflecting better the total extent of injured myocardium. ${ }^{8}$ These regions with impaired innervation may be viable and hypersensitive to catecholamine, resulting in increased automaticity and enhanced triggering and it has been demonstrated that the presence of trigger zone can lead to ventricular arrhythmia and sudden cardiac death. ${ }^{9}$ In this clinical context, it is important to find and quantify the regions of mismatch between infarcted and denervated myocardial region in order to provide appropriate medical or surgical intervention.

\section{DUAL ISOTOPE MYOCARDIAL IMAGING}

The advantages of dual isotope imaging with simultaneous acquisition techniques have been provided in different studies as compared to dual isotope techniques using separate acquisition ${ }^{10,11}$ offering the possibility to reduce total imaging time, to improve patient comfort and also to obtain automatically perfected registered images in both space and time as opposed to ones acquired sequentially, where imaging registration may be necessary. However, performing these simultaneous techniques by standard cameras with a sodium iodide crystal and a typical energy resolution of $10 \%$ can lead to cross-contamination between the two photopeaks of the radiotracers, with consequent artifacts and reduction of image quality. Moreover, the potentially large patient radiation dose resulting from using two radiopharmaceuticals with standard camera can represent another important limitation. Advances in imaging technologies with the introduction of new dedicated cardiac cameras with cadmium-zinc-telluride (CZT) semiconductors, characterized by a higher photon 
sensitivity and spatial resolution than standard cameras, have offered the possibility to overcome these limitations allowing the assessment of myocardial perfusion and innervation in a single imaging session with a limited radiation exposure. In fact, Kacperski et al ${ }^{12}$ evaluating a method of correcting self-scatter and crosstalk effects in simultaneous 99mTechnetium (Tc)/ 201Thallium $(\mathrm{Tl})$ stress/rest MPI showed that although down-scatter of the $140 \mathrm{keV} 99 \mathrm{mTc}$ photons into the main $201 \mathrm{Tl}$ window $(70 \mathrm{keV})$ should be a major problem, the much better energy resolution of CZT detectors, as compared with the conventional anger camera, allows the use of narrower energy windows, thereby reducing down-scatter contribution and effective scatter correction. The iterative deconvolution method developed has been used for the calibrations of dynamic single-photon emission computed tomography (DSPECT) and is a hybrid between the triple energy window method and scatter modeling based on spatial and spectral distribution of projection counts in multiple selected energy windows. ${ }^{12}$ Moreover, taking advantage of the superior energy resolution of CZT camera a modification of the model-based correction algorithm by Kacperski et al ${ }^{12}$ has been proposed to obtain a better separation of the two photopeaks. ${ }^{13}$ In a clinical application performed in 27 patients with a history of ischaemic heart disease, Ben-Haim et $\mathrm{al}^{14}$ demonstrated comparable diagnostic performance and image quality between simultaneous dual-radionuclide $99 \mathrm{mTc}$ stress and $201 \mathrm{Tl}$ rest MPI using a solid state camera and separate dual acquisition using conventional camera. Few studies using these new generation cameras assessed combined myocardial perfusion and sympathetic innervation imaging. ${ }^{15,16}$ Gimelli et $\mathrm{al}^{15}$ investigated the relationships between the measures of regional myocardial adrenergic innervation heterogeneity, cardiac perfusion, and mechanical function with a dedicated CZT camera and low-dose early and delayed 123I-MIBG (74-111 MBq) acquisition followed by rest gated $99 \mathrm{~m}$-Tc-tetrofosmin (222-259 MBq) in a single day protocol without scatter correction and showing that early 123I-MIBG defect score correlated with indicators of myocardial perfusion and functional data in patients with ischemic heart disease. In a recent study, Bellevre et $\mathrm{al}^{16}$ evaluated simultaneous dual isotope (235 MBq123I-MIBG/753 MBq 99mTc-tetrofosmin) acquisition protocol, comparing the delayed MIBG image by traditional camera to combine delayed MIBG/tetrofosmin by CZT camera in patients with heart failure demonstrating a good concordance and agreement. In this feasibility study, simultaneous dual isotope protocol provided a clearer tetrofosmin images and perfect registration to define the heart contours on the tetrofosmin images whit an accurate measurements of 123I-
MIBG heart uptake. List-mode acquisition permitted the retrospective selection of $99 \mathrm{mTc}$ and $123 \mathrm{I}$ energy windows and phantom data (acquired separately for the two radionuclides) showed that $99 \mathrm{mTc}$ crosstalk into the 123I window was negligible when performing a simultaneous acquisition. ${ }^{16}$ In the current issue of the Journal, D'estanque et al $^{17}$ evaluated simultaneous dual isotope myocardial imaging by DSPECT using $92 \mathrm{MBq}$ $201 \mathrm{Tl} / 185 \mathrm{MBq}$ 123I-MIBG in 69 patients revascularized for myocardial infarction. The authors, using a semiquantitative evaluation on a standardized 17 segmentation model, evaluated perfusion and innervation summed rest scores on both early and delayed dual isotope images, with and without scatter correction. Trigger summed scores were than obtained by summing among 17 segmental scores in the delayed innervation images those scores corresponding to viable myocardium on early and delayed dual isotope viability studies. The indices of scar were assessed and compared with the results of the magnetic resonance imaging (MRI) or transthoracic echography as gold-standard methodologies. The authors trying to optimize the protocol for the assessment of trigger zone using a simultaneous evaluation of perfusion and innervation, wondering if the delayed acquisition of innervation images and the scatter correction for thallium studies are necessary.

\section{CURRENT KNOWLEDGE TO GIVE THE ANSWER}

The first challenge of the results presented by D'estanque et $\mathrm{al}^{17}$ is that the delayed acquisitions provided the best mapping of segmental myocardial sympathetic denervation useful to define the trigger zones. This is an interesting point considering that later timing (3.5 hours) for the innervation activity by MIBG correlates with optimal timing to assess viability by thallium in this study. Late HMR was indicated as the most powerful independent predictor of cardiac mortality in patients with ischemic cardiomyopathy. ${ }^{4}$ Measures of delayed 123I-MIBG distribution are demonstrated as more accurate indicators of neuronal activity considering that late heart-to-mediastinum ratio (HMR) is an expression of overall cardiac presynaptic innervation and function as determined both by an initial MIBG uptake and by washout rate, which is regulated by central stimulation, norepinephrine release, reuptake, and spillover. ${ }^{18}$ Using an evaluation of late HMR has been clearly demonstrated the incremental benefit of the combined assessment of myocardial perfusion and sympathetic nerve function for appropriate risk stratification of patients who are likely to need of implantable cardioverter defibrillator. ${ }^{18}$ Only a minority of data using a dual isotope (123I-MIBG/99mTc) tetrofosmin protocol evaluated delayed MIBG images 
using a segmental automatic quantification to assess trigger zone in heart failure patients. ${ }^{19,20}$ The authors reported as new knowledge gained that automated quantitation of delayed myocardial MIBG tomographic imaging in a dual isotope protocol can provide a useful supplement to identify patient with atypical patterns of both innervation and perfusion uptake. The study by D'estanque et $\mathrm{al}^{17}$ is the first clinical experience to evaluate trigger zone in a simultaneous dual isotope 201Tl/123I-MIBG study by CZT camera using the same criteria of automatic quantification in each segment on both perfusion and innervation, by a five-point model depending on mean segmental activity in a segmentation model (17 segments) standardized also for MRI studies performed in $48 \%$ of the overall patients.

Another challenge in the study by D'estanque et $\mathrm{al}^{17}$ was that the authors found a significative difference in trigger zone values between imaging with and without scatter correction. In particular the values of summed rest score and necrosis surface were significantly higher in studies with scatter correction. Accordingly, there were significantly fewer trigger zones in the scatter corrected studies than in uncorrected studies. The extent of necrosis with scatter correction was correlated to the extent obtained with the gold-standard methodologies. The study by D'estanque et al $^{17}$ used in 33 patients contrast-enhanced MRI as gold standard to assess myocardial scar tissue. MRI has been demonstrated having the highest spatial resolution and signal-to-image noise ratio for assessment of the extent and characteristics of scar tissue. Moreover, definition of different signal intensity thresholds on contrast-enhanced MRI data permits differentiation and quantification of the core infarct zone and the peri-infarct or border zone. The extent of peri-infarct zone by MRI in particular has been demonstrated as a powerful predictor of cardiac death and ventricular arrhythmias and the only independent predictor of appropriate ICD therapies. ${ }^{21,22}$ Moreover, previous studies have demonstrated that contrast-enhanced MRI and myocardial perfusion scintigraphy were significantly correlated for infarct size and patients with a significant infarct border zone on MRI showed a significantly larger innervation/perfusion mismatch on 123I-MIBG/perfusion scintigraphy. ${ }^{23}$ The analysis performed by D'estanque et al ${ }^{17}$ using 17 segmental model among perfusion imaging, innervation imaging and MRI lead to a good correspondence in the interpretation of the perfusion-innervation mismatch areas. It remains to consider that not overall patient population has been analyzed with the opportune gold standard by MRI not excluding a possible underestimation of myocardial viability in patients evaluated by echocardiography without dobutamine infusion as gold standard. Thus, further studies are needed to compare the extent of infarct and peri-infarct zones by perfusion and innervation imaging modalities using MRI as gold standard considering that the extent and severity of myocardial dysinnervation remain important but location of dysinnervation remains the most important parameter. Finally, it should also be considered that CZT camera permits potentiality to use lower doses than the ones used by D'estanque et al. ${ }^{17}$ In fact, low-doses by CZT have been demonstrated to be $1.1 \mathrm{MBq} / \mathrm{kg}$ and $74-$ $111 \mathrm{MBq}$ for thallium and MIBG, respectively. ${ }^{15,24} \mathrm{~A}$ potential major reduction of doses could improve the results considering that a decrease of down-scatter contribution from 123I to 201Tl window can be obtained due to higher photon count with semiconductor camera.

Despite at this moment, it is not possible to give a final answer because further studies are needed to clarify how to use all the available instruments to define trigger zone with the new generation cameras, the findings observed by D'estanque et al are important to promote the use of simultaneous dual isotope CZT imaging to improve our knowledge on the relationship between perfusion, innervation, and function. Moreover, considering that quantification of image findings has been and will continue to be crucially important in the assessment of patients with cardiovascular disease further improvements of our acknowledgements are necessary to better clarify the necessity of scatter correction in a low-dose CZT dual isotope protocol according to the combined isotopes and cameras used.

\section{References}

1. Stone GW, Selker HP, Thiele H, Patel MR, Udelson JE, Ohman EM, et al. Relationship between infarct size and outcomes following primary PCI: Patient-level analysis from 10 randomized trials. J Am Coll Cardiol 2016;67:1674-83.

2. Schmidt A, Azevedo CF, Cheng A, Gupta SN, Bluemke DA, Foo $\mathrm{TK}$, et al. Infarct tissue heterogeneity by magnetic resonance imaging identifies enhanced cardiac arrhythmia susceptibility in patients with left ventricular dysfunction. Circulation 2007;115: 2006-14.

3. Underwood SR, Anagnostopoulos C, Cerqueira M, Ell PJ, Flint EJ, Harbinson M, et al. Myocardial perfusion scintigraphy: the evidence. Eur J Nucl Med Mol Imaging 2004;31:261-91.

4. Verberne HJ, Brewster LM, Somsen GA, van Eck-Smit BL. Prognostic value of myocardial 123I-metaiodobenzylguanidine (MIBG) parameters in patients with heart failure: A systematic review. Eur Heart J 2008;29:1147-59.

5. Petretta M, Pellegrino T, Cuocolo A. Cardiac neuronal imaging with ${ }^{123}$ I-meta-iodobenzylguanidine in heart failure: implications of endpoint selection and quantitative analysis on clinical decisions. Eur J Nucl Med Mol Imaging 2014;41:1663-5.

6. Marini C, Giorgetti A, Gimelli A, Kusch A, Sereni N, L'abbate A, et al. Extension of myocardial necrosis differently affects MIBG retention in heart failure caused by ischaemic heart disease or by dilated cardiomyopathy. Eur J Nucl Med Mol Imaging 2005;32:682-8 
7. Du Y, Bhattacharya M, Frey EC. Simultaneous Tc-99m/I-123 dual-radionuclide myocardial perfusion/innervation imaging using Siemens IQ-SPECT with SMARTZOOM collimator. Phys Med Biol 2014;59:2813-28.

8. Matsunari I, Schricke U, Bengel FM, Haase HU, Barthel P, Schmidt G, et al. Extent of cardiac sympathetic neuronal damage is determined by the area of ischemia in patients with acute coronary syndromes. Circulation 2000;101:2579-85.

9. Boogers MJ, Borleffs CJ, Henneman MM, van Bommel RJ, van Ramshorst J, Boersma E, et al. Cardiac sympathetic denervation assessed with 123-iodine metaiodobenzylguanidine imaging predicts ventricular arrhythmias in implantable cardioverterdefibrillator patients. J Am Coll Cardiol 2010;55:2769-77.

10. Weinmann P, Faraggi M, Moretti JL, Hannequin P. Clinical validation of simultaneous dual-isotope myocardial scintigraphy. Eur J Nucl Med Mol Imaging 2003;30:25-31.

11. Kumita S, Cho K, Nakajo H, Toba M, Kijima T, Mizumura S, Kumazaki T, et al. Simultaneous assessment of Tc-99m-sestamibi and I-123-BMIPP myocardial distribution in patients with myocardial infarction: evaluation of left ventricular function with ECG-gated myocardial SPECT. Ann Nucl Med 2000;14:453-9.

12. Kacperski K, Erlandsson K, Ben-Haim S, Hutton BF. Iterative deconvolution of simultaneous $99 \mathrm{mTc}$ and $201 \mathrm{Tl}$ projection data measured on a CdZnTe-based cardiac SPECT scanner. Phys Med Biol 2011;56:1397-414.

13. Holstensson M, Erlandsson K, Poludniowski G, Ben-Haim S, Hutton BF. Model-based correction for scatter and tailing effects in simultaneous 99mTc and 123I imaging for a CdZnTe cardiac SPECT camera. Phys Med Biol 2015;60:3045-63.

14. Ben-Haim S, Kacperski K, Hain S, Van Gramberg D, Hutton BF, Erlandsson K, et al. Simultaneous dual-radionuclide myocardial perfusion imaging with a solid-state dedicated cardiac camera. Eur J Nucl Med Mol Imaging 2010;37:1710-21.

15. Gimelli A, Liga R, Giorgetti A, Genovesi D, Marzullo P. Assessment of myocardial adrenergic innervation with a solidstate dedicated cardiac cadmium-zinc-telluride camera: first clinical experience. Eur Heart J Cardiovasc Imaging 2014;15:575-85.

16. Bellevre D, Manrique A, Legallois D, Bross S, Baavour R, Roth N, et al. First determination of the heart-to-mediastinum ratio using cardiac dual isotope $\left({ }^{123} \mathrm{I}-\mathrm{MIBG} /{ }^{99} \mathrm{mTc}\right.$-tetrofosmin) CZT imaging in patients with heart failure: the ADRECARD study. Eur J Nucl Med Mol Imaging 2015;42:1912-9.
17. D'estanque E, Hedon C, Lattuca B, Bourdon A, Benkiran M, Verd A, et al. Optimization of a simultaneous dual isotope 201Tl/ 123IMIBG myocardial SPECT imaging protocol with a CZT camera for trigger zone assessment after myocardial infarction for routine clinical settings: are delayed acquisition and scatter correction necessary?. J Nucl Cardiol 2016. doi:10.1007/s12350-016-0524-1.

18. Nishisato $K$, Hashimoto A, Nakata $T$, Doi $T$, Yamamoto $H$, Nagahara D, et al. Impaired cardiac sympathetic innervation and myocardial perfusion are related to lethal arrhythmia: Quantification of cardiac tracers in patients with ICDs. J Nucl Med 2010;51:1241-9.

19. Zhou Y, Zhou W, Folks RD, Manatunga DN, Jacobson AF, Bax $\mathrm{JJ}$, et al. I-123 mIBG and Tc-99m myocardial SPECT imaging to predict inducibility of ventricular arrhythmia on electrophysiology testing: a retrospective analysis. J Nucl Cardiol 2014;21:913-20.

20. Clements IP, Garcia EV, Chen J, Folks RD, Butler J, Jacobson AF. Quantitative iodine-123-metaiodobenzylguanidine (MIBG) SPECT imaging in heart failure with left ventricular systolic dysfunction: Development and validation of automated procedures in conjunction with technetium-99m tetrofosmin myocardial perfusion SPECT. J Nucl Cardiol 2016;23:425-35.

21. Yan AT, Shayne AJ, Brown KA, Gupta SN, Chan CW, Luu TM, et al. Characterization of the peri-infarct zone by contrast-enhanced cardiac magnetic resonance imaging is a powerful predictor of post-myocardial infarction mortality. Circulation 2006; 114:32-9.

22. Roes SD, Borleffs CJ, van der Geest RJ, Westenberg JJ, Marsan NA, Kaandorp TA, et al. Infarct tissue heterogeneity assessed with contrast-enhanced MRI predicts spontaneous ventricular arrhythmia in patients with ischemic cardiomyopathy and implantable cardioverter-defibrillator. Circ Cardiovasc Imaging 2009;2:183-90.

23. Ibrahim T, Nekolla SG, Hörnke M, Bülow HP, Dirschinger J, Schömig A, et al. Quantitative measurement of infarct size by contrast-enhanced magnetic resonance imaging early after acute myocardial infarction: comparison with single-photon emission tomography using Tc99m-sestamibi. J Am Coll Cardiol 2005;45:544-52.

24. Songy B, Guernou M, Lussato D, Queneau M, Geronazzo R. Lowdose thallium-201 protocol with a cadmium-zinc-telluride cardiac camera. Nucl Med Commun 2012;33:464-9. 